Article

\title{
The Quantum Nature of Lorentz Invariance
}

\author{
Richard Kerner
}

Laboratoire de Physique Théorique de la Matière Condensée, Sorbonne-Université, 4 Place Jussieu, 75005 Paris, France; richard.kerner@upmc.fr

Received: 21 November 2018; Accepted: 17 December 2018; Published: 20 December 2018

\begin{abstract}
If the reality underlying classical physics is quantum in nature, then it is reasonable to assume that the transformations of fields, currents, energy, and momentum observed macroscopically are the result of averaging of symmetry groups acting in the Hilbert space of quantum states of elementary constituents of which classical material bodies are formed. We show how Pauli's exclusion principle based on the discrete $Z_{2}$ symmetry group generates the $S L(2, \mathrm{C})$ symmetry of the space of states of an electron endowed with spin. Then, we generalize this reasoning in the case of quark colors and the corresponding $Z_{3}$ symmetry. A ternary generalization of Dirac's equation is proposed, leading to self-confined quarks states. It is shown how certain cubic or quadratic combinations can form freely-propagating entangled states. The entire symmetry of the standard model, $S U(2) \times U(1) \times S U(3)$, is naturally derived, as well.
\end{abstract}

Keywords: quark model; $Z_{3}$-graded algebras; color dynamics; Dirac's equation; Loretnz invariance

\section{Introduction}

Since the advent of quantum physics, great care has been taken to demonstrate that all quantum phenomena, when averaged over a great number of events and measurements performed on the atomic scale, lead to the well-known classical limits. The existence of such a limit was considered as one of the cornerstones of the proper formulation of quantum mechanics, and Bohr made the "correspondence principle" a central point in his construction of quantum theory of matter.

On the other hand, it can be stated now without any doubt left that quantum physics is primordial with respect to other observable phenomena perceived by us on the classical level. Therefore, also the fundamental symmetries of classical physics take their source in the underlying symmetries of the deeper quantum reality.

Upon closer scrutiny, the totality of information we receive from our surrounding physical reality is conveyed exclusively by photons (or the electromagnetic forces). Even the recent detection of gravitational waves by the LIGO experimental device was based on the observation of photons in a giant interferometer. All our senses, including auditive and tactile impressions, are conveyed by electromagnetic forces exclusively, even when they are screened as in the van der Waals or Lennard-Jones potentials.

The electromagnetic field, as described by Maxwell's equations, is intrinsically Lorentz-invariant. The same is true for the relativistic Dirac equation of the electron, realized by the operators containing $4 \times 4$ matrices $\gamma^{\mu}$ acting on four-component spinors. The Dirac matrices transform as a Lorentz vector, whereas the spinors undergo the same transformation realized by the corresponding matrices from the $S L(2, \mathrm{C})$ group, which is a double covering of the Lorentz group. It is no wonder that the image of the external world produced with the Lorentz covariant physical entities bears the imprint of their intrinsic symmetry properties.

It seems therefore quite natural to attempt a derivation of all geometric properties from fundamental symmetries and interactions characterizing matter's most fundamental building blocks. 
It is known that many of those properties do not require any mention of space and time on the quantum mechanical level, as was demonstrated by Born and Heisenberg [1] in their version of matrix mechanics, or by von Neumann's formulation of quantum theory in terms of the $C^{*}$ algebras [2]. The non-commutative geometry is another example of the formulation of space-time relationships in purely algebraic terms [3,4].

Quantum mechanics started as a non-relativistic theory, but very soon, its relativistic generalization was created. As an immediate consequence, the wave functions of the Schroedinger picture were required to belong to one of the linear representations of the Lorentz group, which means that they must satisfy the following covariance principle:

$$
\psi^{\prime}\left(x^{\prime}\right)=\psi^{\prime}(\Lambda(x))=S(\Lambda) \psi(x) .
$$

The nature of the representation $S(\Lambda)$ should determine the character of the field considered: spinorial, vectorial, tensorial, etc. As in many other fundamental relations, the seemingly simple Equation (1) creates a bridge between the two totally different realms: the space-time accessible via classical macroscopic observations and the Hilbert space of quantum states. It can be interpreted in two opposite ways, depending on which side we consider as the cause and which one as the consequence. However, it should be stressed that the space-time variables are not conserved quantities like energy and momentum, whose values can be really determined in standard detectors and measuring devices. In this sense, the variables $t, \mathbf{x}$ form a dual space with respect to the linear space of $E$, $\mathbf{p}$, which are the only real conserved parameters given experimentally.

A crucial question arises then: is the macroscopically-observed Lorentz symmetry imposed on the micro-world of quantum physics, or maybe it is already present as the symmetry of quantum states, and then implemented and extended to the macroscopic world in the classical limit? In such a case, the new covariance principle must be written as follows:

$$
j^{\mu^{\prime}}(S(\psi))=j^{\mu^{\prime}}\left(\psi^{\prime}\right)=\Lambda_{\mu}^{\mu^{\prime}}(S) j^{\mu}(\psi),
$$

In the above formula $j^{\mu}=\bar{\psi} \gamma^{\mu} \psi$ is the Dirac current, and $\psi$ is the electron wave function.

Following the analysis of this causal chain, it seems more appropriate to write the same transformations with $\Lambda$ depending on $S$ :

$$
\begin{gathered}
\psi^{\prime}\left(x^{\mu^{\prime}}\right)=S \psi\left(x^{v}\right)=\psi^{\prime}\left(\Lambda_{v}^{\mu^{\prime}}(S) x^{\nu}\right) \\
x^{\mu^{\prime}}(S \psi, \bar{\psi} \bar{S})=\Lambda_{\mu}^{\mu^{\prime}}(S) x^{\mu}(\psi, \bar{\psi}) .
\end{gathered}
$$

This form of the same relation suggests that it is the transition from one quantum state to another, represented by a unitary transformation $S$ that is the primary cause implying the transformation of observed quantities such as the electric four-current and, as a consequence, the apparent transformations of time and space intervals measured with classical physical devices. Similar ideas on the deep origin of symmetries in physics can be found also in Ref. [5].

Although mathematically, the two formulations are equivalent, it seems more plausible that the Lorentz group resulting from the averaging of the action of the $S L(2, \mathbf{C})$ in the Hilbert space of states contains less information than the original double-valued representation, which is a consequence of the particle-anti-particle symmetry, rather than the other way round.

In the present paper, we shall draw physical consequences from this approach, concerning the strong interactions in the first place. However, before considering these, which describe the forces conveyed by gluons and acting among quarks constituting hadrons, let us first see how the Lorentz group appears through the $S L(2, \mathrm{C})$ group action on fermions, in particular on the electron states. 


\section{From Pauli to Lorentz}

The exclusion principle, due to Pauli's pioneering work [6], is one of the most important cornerstones of quantum physics [6]. Stipulating that two identical fermions cannot occupy the same quantum state at once, it explains the structure of atoms and the periodic table of elements and guarantees the stability of matter, preventing its collapse, as suggested by Ehrenfest and proven later by Dyson $[7,8]$. The link between the exclusion principle and particle's spin, known as the "spin-and-statistic theorem", is one of the deepest results in quantum field theory [9].

In the case of the electron, it was discovered that this smallest and indivisible carrier of electric charge is also endowed with a magnetic moment and the intrinsic angular momentum, called "the spin", which can take on exclusively two values. This explains the structure of electronic shells in atoms, where for a given main quantum number $n$, there exist two $n^{2}$ different states.

The $n^{2}$ states come from the possibility for the electron with the energy state given by $n$ to take on different magnetic number states, labeled by the magnetic number $m$ taking values from $-l$ to $+l$, including zero. With two exclusive possibilities for spin, the total number of states in all shells for a given $n$ will be two $n^{2}$, conformally to the observations.

In purely algebraical terms, Pauli's exclusion principle amounts to the anti-symmetry of wave functions describing two coexisting particle states. The easiest way to see how the principle works is to apply Dirac's formalism in which wave functions of particles in a given state are obtained as products between the "bra" and "ket" vectors [10].

The most important statement concerning the spin of the electron is that two electrons cannot occupy the same state, with all quantum numbers equal, including spin. If all quantum numbers except the spin are equal for two electrons, their spins must be opposed; in other words, if the two opposite spin states are created by:

$$
a_{1}|0>=| 1>, \quad a_{2}|0>=| 2>,
$$

Both creation operators are supposed to be nilpotent,

$$
a_{1} a_{1}|0>=| 1,1>=0 \quad a_{2} a_{2}|0>=| 2,2>=0,
$$

thus making impossible the coexistence of two electrons with the same state of spin.

Forming an arbitrary linear superposition of two mutually-exclusive states, we get:

$$
|u>=\lambda| 1>+\mu \mid 2>
$$

Such a state is created by the corresponding linear combination of creation operators:

$$
u=\lambda a_{1}+\mu a_{2}
$$

This operator, creating a possible state of an electron, must be also nilpotent, so that:

$$
u^{2}|0>=u| u>=\mid u, u>=0 .
$$

Writing explicitly the square of the linear combination $u=\lambda a_{1}+\mu a_{2}$, we get:

$$
u^{2}=\left(\lambda a_{1}+\mu a_{2}\right)^{2}=\lambda^{2} a_{1}^{2}+\lambda \mu a_{1} a_{2}+\mu \lambda a_{2} a_{1}+\mu^{2} a_{2}^{2} .
$$

This expression must vanish according to the assumption (9). As both operators $a_{1}$ and $a_{2}$ were supposed to be nilpotent, what remains is:

$$
\lambda \mu\left(a_{1} a_{2}+a_{2} a_{1}\right)=0, \quad \rightarrow \quad a_{1} a_{2}=-a_{2} a_{1}
$$


(the numbers $\mu$ and $\lambda$ commute, so that $\mu \lambda=\lambda \mu$ ).

Let us introduce a contravariant tensor:

$$
\epsilon^{\alpha \beta}, \quad \alpha, \beta=1,2 .
$$

Contracting with two creation operators, we can write:

$$
\epsilon^{\alpha \beta} a_{\alpha} a_{\beta}=\frac{1}{2}\left[\epsilon^{\alpha \beta} a_{\alpha} a_{\beta}+\epsilon^{\beta \alpha} a_{\beta} a_{\alpha}\right],
$$

However, $a_{\alpha} a_{\beta}=-a_{\beta} a_{\alpha}$; therefore:

$$
\epsilon^{\alpha \beta} a_{\alpha} a_{\beta}=\frac{1}{2}\left[\epsilon^{\alpha \beta}-\epsilon^{\beta \alpha}\right] a_{\alpha} a_{\beta},
$$

from which follows, taking into account that there is only one independent non-vanishing combination, $a_{1} a_{2}$, we get:

$$
\epsilon^{\alpha \beta}=\frac{1}{2}\left[\epsilon^{\alpha \beta}-\epsilon^{\beta \alpha}\right],
$$

and consequently,

$$
\epsilon^{\alpha \beta}=-\epsilon^{\beta \alpha} \rightarrow \epsilon^{11}=\epsilon^{22}=0, \epsilon^{12}=-\epsilon^{21} .
$$

There exists a natural dual algebra of contravariant entities $\xi^{\alpha}, \alpha, \beta=1,2$ satisfying the anti-commutation relations:

$$
\xi^{\alpha} \xi^{\beta}=-\xi^{\beta} \xi^{\alpha},
$$

with a corresponding two-form:

$$
\epsilon_{\alpha \beta}=-\epsilon_{\beta \alpha} .
$$

The two-form $\epsilon_{\alpha \beta}$ can be considered as inverse to $\epsilon^{\alpha \beta}$ because one has:

$$
\epsilon_{\alpha \beta} \epsilon^{\gamma \beta}=\delta_{\alpha}^{\gamma}
$$

Note the order of indices being contracted: with a different choice, we would get the identity matrix with the minus sign on the right.

Now, if we require that Pauli's principle must apply independently of the choice of a basis in Hilbert space, i.e., that after a linear transformation, we get:

$$
\epsilon^{\alpha^{\prime} \beta^{\prime}}=S_{\alpha}^{\alpha^{\prime}} S_{\beta}^{\beta^{\prime}} \epsilon^{\alpha \beta}=,=-\epsilon^{\beta^{\prime} \alpha^{\prime}} \text {, with } \epsilon^{1^{\prime} 2^{\prime}}=1,
$$

then the $2 \times 2$ complex matrix $S_{\alpha}^{\alpha^{\prime}}$ must have the determinant equal to one, because then we must have:

$$
\epsilon^{1^{\prime} 2^{\prime}}=S_{\alpha}^{1^{\prime}} S_{\beta}^{2^{\prime}} \epsilon^{\alpha \beta}=S_{1}^{1^{\prime}} S_{2}^{2^{\prime}}-S_{2}^{1^{\prime}} S_{1}^{2^{\prime}}=\operatorname{det} S=1,
$$

which defines the $S L(2, \mathrm{C})$ group.

Now, we have clearly the invariance under the product group $Z_{2} \times Z_{2}$. The first $Z_{2}$ factor is relative to the half-integer dichotomic spin variable, the second $Z_{2}$ group is related to this new universal symmetry, called charge conjugation, stipulating the existence of anti-particles. The four-component complex function $\psi$ is a column composed of two two-component spinors, $\xi_{\alpha}$ and $\chi_{\dot{\beta}}$, which are supposed to transform under two non-equivalent representations of the $S L(2, \mathrm{C})$ group:

$$
\xi_{\alpha^{\prime}}=S_{\alpha^{\prime}}^{\alpha} \xi_{\alpha,} \chi_{\dot{\beta}^{\prime}}=S_{\dot{\beta}^{\prime}}^{\dot{\beta}} \chi_{\dot{\beta}^{\prime}}
$$


The electric charge conservation is equivalent to the vanishing of the four-divergence of $j^{\mu}$ :

$$
\partial_{\mu} j^{\mu}=\left(\partial_{\mu} \psi^{\dagger} \gamma^{\mu}\right) \psi+\psi^{\dagger}\left(\gamma^{\mu} \partial_{\mu} \psi\right)=0,
$$

from which we infer that this condition will be satisfied if we have:

$$
\partial_{\mu} \psi^{\dagger} \gamma^{\mu}=-m \psi^{\dagger} \text { and } \gamma^{\mu} \partial_{\mu} \psi=m \psi \text {, }
$$

which coincides with the Dirac equation [11].

\section{Elementary Properties of Quarks}

According to present knowledge, the ultimate indivisible and indestructible constituents of matter, called atoms by ancient Greeks, are in fact the elementary particles named quarks, carrying fractional electric charges and fractional baryonic numbers, two features that appear to be indestructible and conserved under any circumstances [12-14].

What can be regarded as an extremely strange feature is the fact that freely-propagating quarks have never been directly observed. However, the deep inelastic scattering experiments in which the electrons with extremely high energies penetrate inside the nucleons and scatter on quarks reveal the presence of almost point-like particles, which seem to be almost free as long as they are confined inside the protons or neutrons. When very high energy nucleons collide, they merge for a short while, and quarks can interact via gluon exchange. The gluons are supposed to be the quanta of a color $S U(3)$ gauge field. Besides, quarks interact also via electroweak gauge fields.

In quantum chromodynamics (QCD), quarks are considered as fermions, endowed with half-integer spin $\frac{1}{2}$. Only three quarks or three anti-quarks can coexist inside a fermionic baryon (respectively, anti-baryon), and a pair quark-antiquark can form a meson with integer spin. Besides, they must belong to different colors, also a three-valued set. Three different colors are needed to combine three quarks into a hadron.

There are two quarks in the first generation, $u$ and $d$ ("up" and "down"), which may be considered as two states of a more general object, just like the proton and neutron in $S U(2)$ symmetry are two isospin components of a nucleon doublet. While there is a place for two quarks in the same $u$-state or $d$-state, there are not not three.

The standard model unifies three elementary forces described by three fundamental gauge fields: the gluon field carrying color charge, the weak nuclear force, and the electromagnetic field, the two latter ones unified previously in the electroweak force model based on the $S U(2) \times U(1)$ gauge group. The electroweak forces interact both with quarks and the leptons.

The first quark model using the $S U(3)$ gauge group was introduced to incorporate the newly-discovered hyperon, a heavy strongly-interacting particle labeled as $\Lambda$, in order to form a triplet $p, n, \Lambda$ subject to the fundamental representation of the $S U(3)$ group [12].

Successful as it could be, it turned out that the $S U(3)$ symmetry mixed up particles with definite baryon number with a particle endowed with a yet unknown quantum feature called "strangeness". Later on, another type of particle, with yet another quantum number "charm", was discovered. The $S U(3)$ symmetry acting on these multiplets was not exact; it was broken by the electroweak interactions.

Incidentally, it became clear that there is an exact $S U(3)$ symmetry acting in the space of colors, a new quantum feature taking on three different values, and not just two like in the case of spin or isospin. By now, the first generations of quarks (the "up" and "down" quarks) and leptons: electrons and neutrinos, have been completed by the next two generations, displaying the same number of degrees of freedom each: the "strange" and "charmed" quarks, the "top" and "bottom" quarks, as well as the mu-meson and mu-neutrino and the "tau" meson and its neutrino. 
The fields that intermediate strong interactions between quarks must carry colors, as well. As they have to change the quark's color after interaction, they must carry two different colors and anti-colors at once, "killing" one and "creating" another. However, the totally "colorless" quadratic combination, which does not lead to color exchange, does not interact with quarks at all. This combination: $R \bar{R}+B \bar{B}+G \bar{G}=0$ must vanish, so that only eight linear combinations of quadratic terms out of nine are independent. These are:

$$
\begin{gathered}
\frac{1}{\sqrt{2}}(R \bar{B}+B \bar{R}), \quad \frac{1}{i \sqrt{2}}(R \bar{B}-B \bar{R}), \quad \frac{1}{\sqrt{2}}(R \bar{G}+G \bar{R}), \\
\frac{1}{i \sqrt{2}}(R \bar{G}-G \bar{R}), \frac{1}{\sqrt{2}}(B \bar{G}+G \bar{B}), \quad \frac{1}{i \sqrt{2}}(B \bar{G}-G \bar{B}), \\
\frac{1}{\sqrt{2}}(R \bar{R}-B \bar{B}), \quad \frac{1}{\sqrt{6}}(R \bar{R}+B \bar{B}-2 G \bar{G}),
\end{gathered}
$$

The above combinations form the basis of eight traceless Gell-Mann $3 \times 3$ matrices, spanning the $S U(3)$ Lie algebra.

One of the most striking features of fundamental fermionic fields that constitute the full collection of elementary particles responsible for all known interactions excepting gravitation is the similarity between quarks and leptons. The three doublets of leptons (with their anti-particles, of course):

$$
\left(\begin{array}{c}
e \\
v_{e}
\end{array}\right),\left(\begin{array}{c}
\mu \\
v_{\mu}
\end{array}\right),\left(\begin{array}{c}
\tau \\
v_{\tau}
\end{array}\right),
$$

can be put into a one-to-one correspondence with three doublets of quarks belonging to three "families":

$$
\left(\begin{array}{l}
u \\
d
\end{array}\right),\left(\begin{array}{l}
s \\
c
\end{array}\right),\left(\begin{array}{l}
t \\
b
\end{array}\right) .
$$

The essential difference being that quarks interact not only by means of the electroweak fields, but also by means of color gauge field intermediating strong forces. Thus, color charge conveys three extra degrees of freedom besides the ones that are common to leptons and quarks. In a sense, we can say that leptons are just "colorless quarks".

In the ultimate theoretical version, all fields are supposed massless, the various masses arising due to the Higgs-Kibble-Goldstone mechanism, which might be different for quarks and for leptons.

Now, the quarks being hidden inside the hadrons-strongly-interacting nucleons and mesons-apparently must also display the Lorentz symmetry, which is lifted to the level of composite hadrons, which behave as half-integer spin particles, which were at first considered as elementary, but nowadays without any doubt are known to be composite ones.

The considerations above suggest that a convenient generalization of Pauli's exclusion principle would be that no three quarks in the same state can be present in a nucleon.

Let us require then the vanishing of wave functions representing the tensor product of three (but not necessarily two) identical states: $\Phi(x, x, x)=0$ for any state $|x\rangle$. Let us consider an arbitrary superposition of three different states, $|x\rangle,|y\rangle$ and $|z\rangle$,

$$
|w>=\alpha| x>+\beta|y>+\gamma| z>
$$

and apply the same criterion, $\Phi(w, w, w)=0$. 
We get then, after developing the tensor products,

$$
\begin{gathered}
\Phi(w, w, w)=\alpha^{3} \Phi(x, x, x)+\beta^{3} \Phi(y, y, y)+\gamma^{3} \Phi(z, z, z) \\
+\alpha^{2} \beta[\Phi(x, x, y)+\Phi(x, y, x)+\Phi(y, x, x)]+\gamma \alpha^{2}[\Phi(x, x, z)+\Phi(x, z, x)+\Phi(z, x, x)] \\
+\alpha \beta^{2}[\Phi(y, y, x)+\Phi(y, x, y)+\Phi(x, y, y)]+\beta^{2} \gamma[\Phi(y, y, z)+\Phi(y, z, y)+\Phi(z, y, y)] \\
+\beta \gamma^{2}[\Phi(y, z, z)+\Phi(z, z, y)+\Phi(z, y, z)]+\gamma^{2} \alpha[\Phi(z, z, x)+\Phi(z, x, z)+\Phi(x, z, z)] \\
+\alpha \beta \gamma[\Phi(x, y, z)+\Phi(y, z, x)+\Phi(z, x, y)+\Phi(z, y, x)+\Phi(y, x, z)+\Phi(x, z, y)]=0 .
\end{gathered}
$$

The terms $\Phi(x, x, x), \Phi(y, y, y)$, and $\Phi(z, z, z)$ do vanish by virtue of the original assumption; in what remains, combinations preceded by various powers of independent numerical coefficients $\alpha, \beta$, and $\gamma$, must vanish separately.

This is achieved if the following $Z_{3}$ symmetry is imposed on our wave functions:

$$
\Phi(x, y, z)=j \Phi(y, z, x)=j^{2} \Phi(z, x, y) .
$$

with $j=e^{\frac{2 \pi i}{3}}, j^{3}=1, j+j^{2}+1=0$. Quite naturally, the complex conjugates of functions $\Phi(x, y, z)$ transform under cyclic permutations of their arguments with $j^{2}=\bar{j}$ replacing $j$ in the above formula

$$
\Psi(x, y, z)=j^{2} \Psi(y, z, x)=j \Psi(z, x, y) .
$$

Inside a hadron, not two, but three quarks in different states (colors) can coexist.

After second quantization, when the fields become operator-valued, an alternative cubic commutation relations seems to be more appropriate: instead of $\Psi^{a} \Psi^{b}=(-1) \Psi^{b} \Psi^{a}$, we can introduce $\theta^{A} \theta^{B} \theta^{C}=j \theta^{B} \theta^{C} \theta^{A}=j^{2} \theta^{C} \theta^{A} \theta^{B}$, with $j=e^{\frac{2 \pi i}{3}}$.

In what follows, we shall explore the consequences of such $Z_{3}$ symmetry imposed not only on quark states resulting from the action of corresponding creation operators, but also on the wave equations that should generalize the classical Dirac equation including color degrees of freedom besides the electric charge and baryonic number.

\section{Ternary $Z_{3}$-Commutation}

Let us now consider the possibility of the generalization of the spin and statistic relationship that would incorporate the action of the $Z_{3}$ symmetry group on quark states. If we admit the similarity between the color degree of freedom and spin, we see that while the half-integer spin of ordinary fermions can take on two distinct values, the color takes on three distinct values. Therefore, the wave functions describing quarks must have at least three components; besides, quarks carry ordinary half-integer spin, which requires two components; therefore, we must arrive to at least six component wave functions. Including the anti-particles, the number of components will grow up to twelve.

On the other hand, besides the three-valued color degree of freedom and half-integer spin, quarks appear in two forms, which in the first generation are "up" and "down" states, denoted symbolically by $u$ and $d$. These states are not as exclusive as the "up" and "down" states of an ordinary fermion like the electron, but can coexist in ternary combinations like $u u d$ (the proton) or $u d d$ (the neutron).

According to the $\mathrm{QCD}$, hadrons obeying Fermi statistics (protons and neutrons, to begin with) are composed of three quarks. This raises a natural question: How do their quantum states respond to permutations between those elementary components? To answer it, we should explore algebraic structures that would privilege cubic or ternary relations, in other words, find appropriate cubic or ternary algebras reflecting the most important properties of quark states.

The symmetric group $S_{3}$ containing all permutations of three different elements is a special case among all symmetry groups $S_{N}$. It is the first in the row to be non-Abelian, and the last one 
that possesses a faithful representation in the complex plane $\mathbf{C}^{1}$. It contains six elements and can be generated with only two elements, corresponding to one cyclic and one odd permutation, e.g., $(a b c) \rightarrow$ $(b c a)$ and $(a b c) \rightarrow(c b a)$. All permutations can be represented as different operations on complex numbers as follows.

Denoting the primitive third root of unity by $j=e^{2 \pi i / 3}$, the cyclic Abelian subgroup $Z_{3}$ contains three elements corresponding to the three cyclic permutations, which can be represented via multiplication by $j, j^{2}$, and $j^{3}=1$ (the identity).

$$
\begin{aligned}
& (A B C) \rightarrow(A B C) \text { represented by } \mathbf{1}, \\
& (A B C) \rightarrow(B C A) \text { represented by } \mathbf{j}, \\
& (A B C) \rightarrow(C A B) \text { represented by } \mathbf{j}^{2},
\end{aligned}
$$

Odd permutations must be represented by idempotents, i.e., by operations whose square is the identity operation. We can make the following choice:

$$
\begin{gathered}
(A B C) \rightarrow(C B A) \text { represented by } z \rightarrow \bar{z} \\
(A B C) \rightarrow(B A C) \text { represented by } z \rightarrow \hat{z} \\
(A B C) \rightarrow(C B A) \text { represented by } z \rightarrow z^{*},
\end{gathered}
$$

where the bar $(\mathbf{z} \rightarrow \overline{\mathbf{z}})$ denotes the complex conjugation, i.e., the reflection in the real line, the hat $\mathbf{z} \rightarrow \hat{\mathbf{z}}$ denotes the reflection in the root $j^{2}$, and the star $\mathbf{z} \rightarrow \mathbf{z}^{*}$ the reflection in the root $j$. The six operations close in a non-Abelian group with six elements. However, if it acts on three objects out of which two are identical, e.g., $(A A B)$, then odd permutations give the same result as even ones, so that only the $Z_{3}$ cyclic Abelian group is represented.

With this in mind, let us define the following $Z_{3}$-graded algebra introducing $N$ generators spanning a linear space over complex numbers, satisfying the following cubic relations $[15,16]$ :

$$
\theta^{A} \theta^{B} \theta^{C}=j \theta^{B} \theta^{C} \theta^{A}=j^{2} \theta^{C} \theta^{A} \theta^{B},
$$

We shall also introduce a similar set of conjugate generators, $\bar{\theta}^{\dot{A}}, \dot{A}, \dot{B}, \ldots=1,2, \ldots, N$, satisfying a similar condition with $j^{2}$ replacing $j$ :

$$
\bar{\theta}^{\dot{A}} \bar{\theta}^{\dot{B}} \bar{\theta}^{\dot{C}}=j^{2} \bar{\theta}^{\dot{B}} \bar{\theta}^{\dot{C}} \bar{\theta}^{\dot{A}}=j \bar{\theta}^{\dot{C}} \bar{\theta}^{\dot{A}} \bar{\theta}^{\dot{B}},
$$

Let us denote this algebra by $\mathcal{A}$.

It can be endowed with a natural $Z_{3}$ grading, considering the generators $\theta^{A}$ as Grade 1 elements, their conjugates $\bar{\theta}^{\dot{A}}$ being of grade 2 . The grades add up modulo three; the products $\theta^{A} \theta^{B}$ span a linear subspace of grade 2 , and the cubic products $\theta^{A} \theta^{B} \theta^{C}$ are of grade 0 .

Similarly, all quadratic expressions in conjugate generators, $\bar{\theta}^{\dot{A}} \bar{\theta}^{\dot{B}}$ are of grade $2+2=4_{\bmod 3}=1$, whereas their cubic products are again of grade 0 , like the cubic products of $\theta^{A \text { 's. }}$.

Combined with the associativity, these cubic relations impose finite dimension on the algebra generated by the $Z_{3}$ graded generators. As a matter of fact, cubic expressions are the highest order that does not vanish identically. The proof is immediate:

$$
\theta^{A} \theta^{B} \theta^{C} \theta^{D}=j \theta^{B} \theta^{C} \theta^{A} \theta^{D}=j^{2} \theta^{B} \theta^{A} \theta^{D} \theta^{C}=j^{3} \theta^{A} \theta^{D} \theta^{B} \theta^{C}=j^{4} \theta^{A} \theta^{B} \theta^{C} \theta^{D},
$$

and because $j^{4}=j \neq 1$, the only solution is $\theta^{A} \theta^{B} \theta^{C} \theta^{D}=0$.

The total dimension of the algebra defined via the cubic relations (21) is equal to $N+N^{2}+\left(N^{3}-\right.$ $N) / 3$ : the $N$ generators of grade 1 , the $N^{2}$ independent products of two generators, and $\left(N^{3}-N\right) / 3$ independent cubic expressions, because the cube of any generator must be zero by virtue of (21), 
and the remaining $N^{3}-N$ ternary products are divided by three, also by virtue of the constitutive relations (21).

The conjugate generators $\bar{\theta}^{\dot{B}}$ span an algebra $\overline{\mathcal{A}}$ isomorphic with $\mathcal{A}$.

If we want the products between the generators $\theta^{A}$ and the conjugate ones $\bar{\theta}^{\dot{B}}$ to be included into the greater algebra spanned by both types of generators, we should consider all possible products, between both types of generators, which will span the resulting algebra $\mathcal{A} \otimes \overline{\mathcal{A}}$.

Let us impose the following commutation relations:

$$
\theta^{A} \bar{\theta}^{\dot{B}}=-j \bar{\theta}^{\dot{B}} \theta^{A}, \quad \bar{\theta}^{\dot{B}} \theta^{A}=-j^{2} \theta^{A} \bar{\theta}^{\dot{B}},
$$

In fact, introducing the "minus" sign, i.e., the multiplication by -1 , we extend the discrete symmetry group acting on our algebra to the product $Z_{3} \times Z_{2}$. It is easy to prove that this product is isomorphic with the cyclic group $Z_{6}$. The choice of commutation relations (24) leads to the anticommutation property between the conjugate cubic monomials:

$$
\left(\theta^{A} \theta^{B} \theta^{C}\right)\left(\bar{\theta}^{\dot{D}} \bar{\theta}^{\dot{E}} \bar{\theta}^{\dot{F}}\right)=-\left(\bar{\theta}^{\dot{D}} \bar{\theta}^{\dot{E}} \bar{\theta}^{\dot{F}}\right)\left(\theta^{A} \theta^{B} \theta^{C}\right)
$$

characteristic for the fermions. This is another hint towards the possibility of forming anti-commuting fermionic variables with cubic combinations of our "quark" operators.

\section{Two-Generator Algebra and Its Invariance Group}

The three quarks constituting hadrons (the latter behaving as fermions) are found in two states, "up" and "down", designed by $u$ and $d$, endowed with fractional electric charges, $+\frac{2}{3}$ for the $u$-quark and $-\frac{1}{3}$ for the $d$-quark. Therefore, the product state $u u d$ will represent a proton (electric charge +1 ), whilst the combination $u d d$ having zero electric charge, represents a neutron. We shall therefore reduce the number of generators of our $Z_{3}$-graded algebra representing quark operators, to the minimal number, i.e., two generators only.

Let us consider the simplest case of cubic algebra with two generators, $A, B, \ldots=1,2$. Its grade 1 component contains just these two elements, $\theta^{1}$ and $\theta^{2}$; its grade 2 component contains four independent products, $\theta^{1} \theta^{1}, \theta^{1} \theta^{2}, \theta^{2} \theta^{1}$, and $\theta^{2} \theta^{2}$. Finally, its grade 0 component (which is a subalgebra) contains the unit element 1 and the two linearly-independent cubic products, $\theta^{1} \theta^{2} \theta^{1}=j \theta^{2} \theta^{1} \theta^{1}=$ $j^{2} \theta^{1} \theta^{1} \theta^{2}$ and $\theta^{2} \theta^{1} \theta^{2}=j \theta^{1} \theta^{2} \theta^{2}=j^{2} \theta^{2} \theta^{2} \theta^{1}$ with two similar independent combinations of conjugate generators $\bar{\theta}^{\dot{A}}$.

Considering now the multilinear forms defined on the algebra $\mathcal{A} \otimes \overline{\mathcal{A}}$. Because only cubic relations are imposed on products in $\mathcal{A}$ and in $\overline{\mathcal{A}}$ and the binary relations on the products of ordinary and conjugate elements, we shall fix our attention on tri-linear and bi-linear forms, conceived as mappings of $\mathcal{A} \otimes \mathcal{A}$ into certain linear spaces over complex numbers. Consider a tri-linear form $\rho_{A B C}^{\alpha}$. We shall call this form $Z_{3}$-invariant if we can write, by virtue of (21):

$$
\begin{gathered}
\rho_{A B C}^{\alpha} \theta^{A} \theta^{B} \theta^{C}=\frac{1}{3}\left[\rho_{A B C}^{\alpha} \theta^{A} \theta^{B} \theta^{C}+\rho_{B C A}^{\alpha} \theta^{B} \theta^{C} \theta^{A}+\rho_{C A B}^{\alpha} \theta^{C} \theta^{A} \theta^{B}\right]= \\
=\frac{1}{3}\left[\rho_{A B C}^{\alpha} \theta^{A} \theta^{B} \theta^{C}+\rho_{B C A}^{\alpha}\left(j^{2} \theta^{A} \theta^{B} \theta^{C}\right)+\rho_{C A B}^{\alpha} j\left(\theta^{A} \theta^{B} \theta^{C}\right)\right],
\end{gathered}
$$

From this, it follows that we should have:

$$
\rho_{A B C}^{\alpha} \theta^{A} \theta^{B} \theta^{C}=\frac{1}{3}\left[\rho_{A B C}^{\alpha}+j^{2} \rho_{B C A}^{\alpha}+j \rho_{C A B}^{\alpha}\right] \theta^{A} \theta^{B} \theta^{C},
$$

from which we get the following properties of the $\rho$-cubic matrices:

$$
\rho_{A B C}^{\alpha}=j^{2} \rho_{B C A}^{\alpha}=j \rho_{C A B}^{\alpha} .
$$


Even in this minimal and discrete case, there are covariant and contravariant indices: the lower and the upper indices display the inverse transformation property. If a given cyclic permutation is represented by a multiplication by $j$ for the upper indices, the same permutation performed on the lower indices is represented by multiplication by the inverse, i.e., $j^{2}$, so that they compensate each other. Similar reasoning leads to the definition of the conjugate forms $\bar{\rho}_{\dot{C} \dot{B} \dot{A}}^{\dot{\alpha}}$ satisfying the relations similar to (27) with $j$ replaced be its conjugate, $j^{2}$ :

$$
\bar{\rho}_{\dot{A} \dot{B} \dot{C}}^{\dot{\alpha}}=j \bar{\rho}_{\dot{B} \dot{C} \dot{A}}^{\dot{\alpha}}=j^{2} \bar{\rho}_{\dot{C} \dot{A} \dot{B}}^{\dot{\alpha}}
$$

In the simplest case of two generators, the $j$-skew-invariant forms have only two independent components:

$$
\rho_{121}^{1}=j \rho_{211}^{1}=j^{2} \rho_{112}^{1}, \quad \rho_{212}^{2}=j \rho_{122}^{2}=j^{2} \rho_{221}^{2},
$$

and we can set:

$$
\rho_{121}^{1}=1, \rho_{211}^{1}=j^{2}, \rho_{112}^{1}=j, \quad \rho_{212}^{2}=1, \rho_{122}^{2}=j^{2}, \rho_{221}^{2}=j .
$$

The constitutive cubic relations between the generators of the $Z_{3}$ graded algebra can be considered as intrinsic if they are conserved after linear transformations with commuting (pure number) coefficients, i.e., if they are independent of the choice of the basis.

Let $U_{A}^{A^{\prime}}$ denote a non-singular $N \times N$ matrix, transforming the generators $\theta^{A}$ into another set of generators, $\theta^{B^{\prime}}=U_{B}^{B^{\prime}} \theta^{B}$.

We are looking for the solution of the covariance condition for the $\rho$-matrices:

$$
S_{\beta}^{\alpha^{\prime}} \rho_{A B C}^{\beta}=U_{A}^{A^{\prime}} U_{B}^{B^{\prime}} U_{C}^{C^{\prime}} \rho_{A^{\prime} B^{\prime} C^{\prime}}^{\alpha^{\prime}}
$$

Now, $\rho_{121}^{1}=1$, and we have two equations corresponding to the choice of values of the index $\alpha^{\prime}$ equal to one or two. For $\alpha^{\prime}=1^{\prime}$, the $\rho$-matrix on the right-hand side is $\rho_{A^{\prime} B^{\prime} C^{\prime}}^{1^{\prime}}$, which has only three components,

$$
\rho_{1^{\prime} 2^{\prime} 1^{\prime}}^{1^{\prime}}=1, \quad \rho_{2^{\prime} 1^{\prime} 1^{\prime}}^{1^{\prime}}=j^{2}, \quad \rho_{1^{\prime} 1^{\prime} 2^{\prime}}^{1^{\prime}}=j,
$$

which leads to the following equation:

$$
S_{1}^{1^{\prime}}=U_{1}^{1^{\prime}} U_{2}^{2^{\prime}} U_{1}^{1^{\prime}}+j^{2} U_{1}^{2^{\prime}} U_{2}^{1^{\prime}} U_{1}^{1^{\prime}}+j U_{1}^{1^{\prime}} U_{2}^{1^{\prime}} U_{1}^{2^{\prime}}=U_{1}^{1^{\prime}}\left(U_{2}^{2^{\prime}} U_{1}^{1^{\prime}}-U_{1}^{2^{\prime}} U_{2}^{1^{\prime}}\right),
$$

because $j^{2}+j=-1$. For the alternative choice $\alpha^{\prime}=2^{\prime}$, the $\rho$-matrix on the right-hand side is $\rho_{A^{\prime} B^{\prime} C^{\prime}}^{2^{\prime}}$ whose three non-vanishing components are:

$$
\rho_{2^{\prime} 1^{\prime} 2^{\prime}}^{2^{\prime}}=1, \quad \rho_{1^{\prime} 2^{\prime} 2^{\prime}}^{2^{\prime}}=j^{2}, \quad \rho_{2^{\prime} 2^{\prime} 1^{\prime}}^{2^{\prime}}=j .
$$

The corresponding equation becomes now:

$$
S_{1}^{2^{\prime}}=U_{1}^{2^{\prime}} U_{2}^{1^{\prime}} U_{1}^{2^{\prime}}+j^{2} U_{1}^{1^{\prime}} U_{2}^{2^{\prime}} U_{1}^{2^{\prime}}+j U_{1}^{2^{\prime}} U_{2}^{2^{\prime}} U_{1}^{1^{\prime}}=U_{1}^{2^{\prime}}\left(U_{2}^{1^{\prime}} U_{1}^{2^{\prime}}-U_{1}^{1^{\prime}} U_{2}^{2^{\prime}}\right)
$$

The remaining two equations are obtained in a similar manner, with the determinant of the $2 \times 2$ matrix $U_{B}^{A^{\prime}}$ appearing on the right-hand side, so that the final result is:

$$
\begin{aligned}
& S_{1}^{1^{\prime}}=U_{1}^{1^{\prime}}[\operatorname{det}(U)], \quad S_{1}^{2^{\prime}}=-U_{1}^{2^{\prime}}[\operatorname{det}(U)], \\
& S_{2}^{1^{\prime}}=-U_{2}^{1^{\prime}}[\operatorname{det}(U)], \quad S_{2}^{2^{\prime}}=U_{2}^{2^{\prime}}[\operatorname{det}(U)] .
\end{aligned}
$$

Taking the determinant of the matrix $S_{\beta}^{\alpha^{\prime}}$, one gets immediately:

$$
\operatorname{det}(S)=[\operatorname{det}(U)]^{3} \text {. }
$$


However, the $U$-matrices on the right-hand side are defined only up to the phase, which is due to the cubic character of the covariance relations (30)-(33), and they can take on three different values: 1 , $j$, or $j^{2}$, i.e., the matrices $j U_{B}^{A^{\prime}}$ or $j^{2} U_{B}^{A^{\prime}}$ satisfy the same relations as the matrices $U_{B}^{A^{\prime}}$ defined above. The determinant of $U$ can take on the values $1, j$, or $j^{2}$ if $\operatorname{det}(S)=1$ However, for the time being, we have no reason yet to impose the unitarity condition. It can be derived from the conditions imposed on the invariance and duality of binary relations between $\theta^{A}$ and their conjugates $\bar{\theta}^{\dot{B}}$. In the Hilbert space of spinors, the $S L(2, \mathrm{C})$ action conserved naturally two anti-symmetric tensors,

$$
\varepsilon_{\alpha \beta} \text { and } \varepsilon_{\dot{\alpha} \dot{\beta}} \text { and their duals } \varepsilon^{\alpha \beta} \text { and } \varepsilon^{\dot{\alpha} \dot{\beta}} \text {. }
$$

Spinorial indices thus can be raised or lowered using these fundamental $\operatorname{SL}(2, \mathrm{C})$ tensors:

$$
\psi_{\beta}=\epsilon_{\alpha \beta} \psi^{\alpha}, \quad \psi^{\dot{\delta}}=\varepsilon^{\dot{\delta} \dot{\beta}} \psi_{\dot{\beta}} .
$$

In the space of quark states, a similar invariant form can be introduced, as well. There is only one alternative: either the Kronecker delta or the anti-symmetric two-form $\varepsilon$. Supposing that our cubic combinations of quark states behave like fermions, there is no choice left: if we want to define the duals of cubic forms $\rho_{A B C}^{\alpha}$ displaying the same symmetry properties, we must impose the covariance principle as follows:

$$
\epsilon_{\alpha \beta} \rho_{A B C}^{\alpha}=\varepsilon_{A D} \varepsilon_{B E} \varepsilon_{C G} \rho_{\beta}^{D E G} \text {. }
$$

The requirement of the invariance of tensor $\varepsilon_{A B}, A, B=1,2$ with respect to the change of basis of quark states leads to the condition $\operatorname{det} U=1$, i.e., again to the $S L(2, \mathrm{C})$ group.

A similar covariance requirement can be formulated with respect to the set of two-forms mapping the quadratic quark-anti-quark combinations into a four-dimensional linear real space. As we already saw, the symmetry (24) imposed on these expressions reduces their number to four. Let us define two quadratic forms, $\pi_{A \dot{B}}^{\mu}$ and its conjugate $\bar{\pi}_{\dot{B} A}^{\mu}$ :

$$
\pi_{A \dot{B}}^{\mu} \theta^{A} \bar{\theta}^{\dot{B}} \text { and } \bar{\pi}_{\dot{B} A}^{\mu} \bar{\theta}^{\dot{B}} \theta^{A} .
$$

The Greek indices $\mu, v \ldots$ run over four values, and we shall label them $0,1,2,3$. The four tensors $\pi_{A \dot{B}}^{\mu}$ and their Hermitian conjugates $\bar{\pi}_{\dot{B} A}^{\mu}$ define a bi-linear mapping from the product of quark and anti-quark cubic algebras into a linear four-dimensional vector space, whose structure is not yet defined. Let us impose the following invariance condition:

$$
\pi_{A \dot{B}}^{\mu} \theta^{A} \bar{\theta}^{\dot{B}}=\bar{\pi}_{\dot{B} A}^{\mu} \bar{\theta}^{\dot{B}} \theta^{A} .
$$

It follows immediately from (24) that:

$$
\pi_{A \dot{B}}^{\mu}=-j^{2} \bar{\pi}_{\dot{B} A}^{\mu}
$$

Such matrices are non-Hermitian, and they can be realized by the following substitution:

$$
\pi_{A \dot{B}}^{\mu}=j^{2} i \sigma_{A \dot{B}^{\prime}}^{\mu} \quad \bar{\pi}_{\dot{B} A}^{\mu}=-j i \sigma_{\dot{B} A}^{\mu}
$$

where $\sigma_{A \dot{B}}^{\mu}$ are the unit 2 matrix for $\mu=0$ and the three Hermitian Pauli matrices for $\mu=1,2,3$.

Again, we want to get the same form of these four matrices in another basis. Knowing that the lower indices $A$ and $\dot{B}$ undergo the transformation with matrices $U_{B}^{A^{\prime}}$ and $\bar{U}_{\dot{B}}^{\dot{A}^{\prime}}$, we demand that there exist some $4 \times 4$ matrices $\Lambda_{v}^{\mu^{\prime}}$ representing the transformation of lower indices by the matrices $U$ and $\bar{U}$ :

$$
\Lambda_{v}^{\mu^{\prime}} \pi_{A \dot{B}}^{v}=U_{A}^{A^{\prime}} \bar{U}_{\dot{B}}^{\dot{B}^{\prime}} \pi_{A^{\prime} \dot{B}^{\prime \prime}}^{\mu^{\prime}}
$$


This defines the vector $(4 \times 4)$ representation of the Lorentz group. The system (39) contains four groups of four equations each, following the choice of values for indices $\mu^{\prime}$ on one side and the indices $A$ and $B$. We shall show explicitly only the first four equations relating the $4 \times 4$ real matrices $\Lambda_{v}^{\mu^{\prime}}$ with the $2 \times 2$ complex matrices $U_{B}^{A^{\prime}}$ and $\bar{U}_{\dot{B}}^{\dot{A}^{\prime}}$, corresponding to the value $\mu^{\prime}=0^{\prime}$ :

$$
\begin{array}{ll}
\Lambda_{0}^{0^{\prime}}+\Lambda_{3}^{0^{\prime}}=U_{1}^{1^{\prime}} \bar{U}_{\dot{1}}^{i^{\prime}}+U_{1}^{2^{\prime}} \bar{U}_{\dot{1}}^{\dot{2}^{\prime}}, & \Lambda_{0}^{0^{\prime}}-\Lambda_{3}^{0^{\prime}}=U_{2}^{1^{\prime}} \bar{U}_{\dot{2}}^{i^{\prime}}+U_{2}^{2^{\prime}} \bar{U}_{2}^{\dot{2}^{\prime}}, \\
\Lambda_{0}^{0^{\prime}}-i \Lambda_{2}^{0^{\prime}}=U_{1}^{1^{\prime}} \bar{U}_{2}^{\dot{1}^{\prime}}+U_{1}^{2^{\prime}} \bar{U}_{\dot{2}}^{\dot{2}^{\prime}}, & \Lambda_{0}^{0^{\prime}}+i \Lambda_{2}^{0^{\prime}}=U_{2}^{1^{\prime}} \bar{U}_{\dot{1}}^{\dot{1}^{\prime}}+U_{2}^{2^{\prime}} \bar{U}_{\dot{1}}^{\dot{2}^{\prime}}
\end{array}
$$

The next three groups of four equations are similar to the above.

With the invariant "spinorial metric" in two complex dimensions, $\varepsilon^{A B}$ and $\varepsilon^{\dot{A} \dot{B}}$, such that $\varepsilon^{12}=$ $-\varepsilon^{21}=1$ and $\varepsilon^{\dot{1} \dot{2}}=-\varepsilon^{2 \dot{1}}$, we can define the contravariant components $\pi^{v} A \dot{B}$. It is easy to show that the Minkowskian space-time metric, invariant under the Lorentz transformations, can be defined as:

$$
g^{\mu \nu}=\frac{1}{2}\left[\pi_{A \dot{B}}^{\mu} \pi^{v} A \dot{B}\right]=\operatorname{diag}(+,-,-,-)
$$

Let us then choose the matrices $S_{\beta}^{\alpha^{\prime}}$ to be the usual spinor representation of the $S L(2, \mathrm{C})$ group, while the matrices $U_{B}^{A^{\prime}}$ will be defined as follows:

$$
U_{1}^{1^{\prime}}=j S_{1}^{1^{\prime}}, U_{2}^{1^{\prime}}=-j S_{2}^{1^{\prime}}, U_{1}^{2^{\prime}}=-j S_{1}^{2^{\prime}}, U_{2}^{2^{\prime}}=j S_{2}^{2^{\prime}},
$$

the determinant of $U$ being equal to $j^{2}$. Obviously, the same reasoning leads to the conjugate cubic representation of the same symmetry group $S L(2, \mathrm{C})$ if we require the covariance of the conjugate tensor:

$$
\bar{\rho}_{\dot{D} \dot{E} \dot{F}}^{\dot{\beta}}=j \bar{\rho}_{\dot{E} \dot{F} \dot{D}}^{\dot{\beta}}=j^{2} \bar{\rho}_{\dot{F} \dot{D} \dot{E}}^{\dot{\beta}}
$$

by imposing the equation similar to (29):

$$
\bar{S}_{\dot{\beta}}^{\dot{\alpha}^{\prime}} \bar{\rho}_{\dot{A} \dot{B} \dot{C}}^{\dot{\beta}}=\bar{\rho}_{\dot{A}^{\prime} \dot{B}^{\prime} \dot{C}^{\prime}}^{\dot{\prime}^{\prime}} \bar{U}_{\dot{A}}^{\dot{A}^{\prime}} \bar{U}_{\dot{B}}^{\dot{B}^{\prime}} \bar{U}_{\dot{C}}^{\dot{C}^{\prime}}
$$

The matrix $\bar{U}$ is the complex conjugate of the matrix $U$, and its determinant is $j$. Moreover, the two-component entities obtained as images of cubic combinations of quarks, $\psi^{\alpha}=\rho_{A B C}^{\alpha} \theta^{A} \theta^{B} \theta^{C}$ and $\bar{\psi}^{\dot{\beta}}=\bar{\rho}_{\dot{D} \dot{E} \dot{F}}^{\dot{\beta}} \bar{\theta}^{\dot{D}} \bar{\theta}^{\dot{E}} \bar{\theta}^{\dot{F}}$ should anti-commute, because their arguments do so, by virtue of (24):

$$
\left(\theta^{A} \theta^{B} \theta^{C}\right)\left(\bar{\theta}^{\dot{D}} \bar{\theta}^{\dot{E}} \bar{\theta}^{\dot{F}}\right)=-\left(\bar{\theta}^{\dot{D}} \bar{\theta}^{\dot{E}} \bar{\theta}^{\dot{F}}\right)\left(\theta^{A} \theta^{B} \theta^{C}\right)
$$

The usual $Z_{2}$ Pauli principle led to the $S L(2, \mathrm{C})$ covering group of the Lorentz group acting on spinors via the spinorial $2 \times 2$ matrix representation. Here, the quarks transform with matrices $U$ (or $\bar{U}$ for the anti-quarks), but these matrices are not unitary: their determinants are equal to $j^{2}$ or $j$, respectively. Therefore, quarks cannot be put on the same footing as classical spinors; they transform under a $Z_{3}$-covering of the Lorentz group. The Dirac spinors should be obtained as the homomorphic image of the tri-linear combination of three quarks (or anti-quarks).

\section{Color Dirac Equation}

Dirac's equation for the electron can be regarded as a system of two equations relating between them two distinct Pauli spinors. The two coupled Pauli equations can be re-written using the matrix notation: 


$$
\left(\begin{array}{ll}
E & 0 \\
0 & E
\end{array}\right)\left(\begin{array}{l}
\psi_{+} \\
\psi_{-}
\end{array}\right)=\left(\begin{array}{cc}
m c^{2} & 0 \\
0 & -m c^{2}
\end{array}\right)\left(\begin{array}{l}
\psi_{+} \\
\psi_{-}
\end{array}\right)+\left(\begin{array}{cc}
0 & c \sigma \mathbf{p} \\
c \sigma \mathbf{p} & 0
\end{array}\right)\left(\begin{array}{l}
\psi_{+} \\
\psi_{-}
\end{array}\right),
$$

where the entries in the energy operator and the mass matrix are in fact $2 \times 2$ identity matrices, as well as the $\sigma$-matrices appearing in the last matrix, so that in reality, the above equation represents the $4 \times 4$ Dirac equation, only in a different basis.

The system of linear equations (44) displays two discrete $Z_{2}$ symmetries: the space reflection consisting of the simultaneous change of directions of spin and momentum, $\sigma \rightarrow-\sigma, \mathbf{p} \rightarrow-\mathbf{p}$, and the particle-antiparticle symmetry realized by the transformation:

$$
m \rightarrow-m, \psi_{+} \rightarrow \psi_{-}, \quad \psi_{-} \rightarrow \psi_{+} .
$$

Our next aim is to extend the $Z_{2} \times Z_{2}$ symmetry by including the $Z_{3}$ group, which will mix not only the two spin states and particles with anti-particles, but also the three colors.

Let us follow the logic that led from Pauli's to Dirac's equation extending it to the colors acted upon by the $Z_{3}$-group. In the expression for the energy operator (i.e., the Hamiltonian), the mass term is positive when acting on particles and acquires a negative sign acting on anti-particles, i.e., it changes sign while intertwining particle-antiparticle components. We shall also assume that the mass term acquires the factor $j$ when we switch from the red component $\varphi$ to the blue component $\chi$, and $j^{2}$ for the green component $\psi$. The momentum operator will be non-diagonal, as in the Dirac equation, systematically intertwining not only particles with antiparticles, but also colors with anti-colors. The system that satisfies all of these assumptions is as follows:

$$
\begin{gathered}
E \varphi_{+}=m c^{2} \varphi_{+}+c \sigma \cdot \mathbf{p} \chi_{-} \\
E \chi_{-}=-j m c^{2} \chi_{-}+c \sigma \cdot \mathbf{p} \psi_{+} \\
E \psi_{+}=j^{2} m c^{2} \psi_{+}+c \sigma \cdot \mathbf{p} \varphi_{-} \\
E \varphi_{-}=-m c^{2} \varphi_{-}+c \sigma \cdot \mathbf{p} \chi_{+} \\
E \chi_{+}=j m c^{2} \chi_{+}+c \sigma \cdot \mathbf{p} \psi_{-} \\
E \psi_{-}=-j^{2} m c^{2} \psi_{-}+c \sigma \cdot \mathbf{p} \varphi_{+}
\end{gathered}
$$

where:

$$
\varphi_{+}=\left(\begin{array}{c}
\varphi_{+}^{1} \\
\varphi_{+}^{2}
\end{array}\right), \varphi_{-}=\left(\begin{array}{c}
\varphi_{-}^{1} \\
\varphi_{-}^{2}
\end{array}\right), \chi_{+}=\left(\begin{array}{c}
\chi_{+}^{1} \\
\chi_{+}^{2}
\end{array}\right), \chi_{-}=\left(\begin{array}{l}
\chi_{-}^{1} \\
\chi_{-}^{2}
\end{array}\right), \psi_{+}=\left(\begin{array}{c}
\psi_{+}^{1} \\
\psi_{+}^{2}
\end{array}\right), \psi_{-}=\left(\begin{array}{l}
\psi_{-}^{1} \\
\psi_{-}^{2}
\end{array}\right),
$$

on which Pauli sigma-matrices act in a natural way.

The energy operator is obviously diagonal, and its action on the spinor-valued column-vector can be represented as a $6 \times 6$ operator valued unit matrix. The mass operator is diagonal, as well, but its elements represent all powers of the sixth root of unity $q=e^{\frac{2 \pi i}{6}}$, which are:

$$
q=-j^{2}, q^{2}=j, q^{3}=-1, q^{2}=j^{2}, q^{5}=-j \text { and } q^{6}=1 .
$$

In the basis in which the column of six "colored" Pauli spinors is arranged as follows:

$$
\left(\varphi_{+}, \chi_{+}, \psi_{+}, \varphi_{-}, \chi_{-}, \psi_{-}\right)
$$


the mass and momentum operators take on the following form:

$$
M=\left(\begin{array}{cccccc}
m & 0 & 0 & 0 & 0 & 0 \\
0 & j m & 0 & 0 & 0 & 0 \\
0 & 0 & j^{2} m & 0 & 0 & 0 \\
0 & 0 & 0 & -m & 0 & 0 \\
0 & 0 & 0 & 0 & -j m & 0 \\
0 & 0 & 0 & 0 & 0 & -j^{2} m
\end{array}\right), \quad P=\left(\begin{array}{cccccc}
0 & 0 & 0 & 0 & \sigma \cdot \mathbf{p} & 0 \\
0 & 0 & 0 & 0 & 0 & \sigma \cdot \mathbf{p} \\
0 & 0 & 0 & \sigma \cdot \mathbf{p} & 0 & 0 \\
0 & \sigma \cdot \mathbf{p} & 0 & 0 & 0 & 0 \\
0 & 0 & \sigma \cdot \mathbf{p} & 0 & 0 & 0 \\
\sigma \cdot \mathbf{p} & 0 & 0 & 0 & 0 & 0
\end{array}\right)
$$

In fact, the dimension of the two matrices $M$ and $P$ displayed above is $12 \times 12$ : all the entries in the first one are proportional to the $2 \times 2$ identity matrix, so that in the definition, one should read $\left(\begin{array}{cc}m & 0 \\ 0 & m\end{array}\right)$ instead of $m,\left(\begin{array}{cc}j m & 0 \\ 0 & j m\end{array}\right)$ instead of $j m$, etc. The entries in the second matrix $P$ contain $2 \times 2$ Pauli's sigma-matrices, so that $P$ is also a $12 \times 12$ matrix. The energy operator $E$ is proportional to the $12 \times 12$ identity matrix.

We can easily check that only even powers of $\sigma$-matrices are proportional to $\mathbb{1}_{2}$, and only the powers of the $3 \times 3$ circulant matrix that are multiplicities of three are proportional to $\mathbb{1}_{3}$; the diagonalization of the system is achieved only at the sixth iteration. The result of the diagonalization is very simple: all the components satisfy the same sixth-order equation,

$$
E^{6} \varphi_{+}=m^{6} c^{12} \varphi_{+}+c^{6}|\mathbf{p}|^{6} \varphi_{+}, \quad E^{6} \varphi_{-}=m^{6} c^{12} \varphi_{-}+c^{6}|\mathbf{p}|^{6} \varphi_{-} .
$$

and similarly for all other components.

The three operators can be expressed in terms of tensor products of matrices of lower dimensions. Let us introduce two following $3 \times 3$ matrices:

$$
B=\left(\begin{array}{ccc}
1 & 0 & 0 \\
0 & j & 0 \\
0 & 0 & j^{2}
\end{array}\right) \text { and } Q_{3}=\left(\begin{array}{ccc}
0 & 1 & 0 \\
0 & 0 & 1 \\
1 & 0 & 0
\end{array}\right)
$$

whose products and powers generate the $U(3)$ Lie group algebra, or the $S U(3)$ algebra if we remove the unit matrix. Then, the $12 \times 12$ matrices $M$ and $P$ can be represented as the following tensor products:

$$
M=m \sigma_{3} \otimes B \otimes \mathbb{1}_{2}, \quad P=\sigma_{1} \otimes Q_{3} \otimes(\sigma \cdot \mathbf{p})
$$

with as usual, $\mathbb{1}_{2}=\left(\begin{array}{ll}1 & 0 \\ 0 & 1\end{array}\right), \quad \sigma_{1}=\left(\begin{array}{ll}0 & 1 \\ 1 & 0\end{array}\right), \quad \sigma_{3}=\left(\begin{array}{cc}1 & 0 \\ 0 & -1\end{array}\right)$.

Let us rewrite the matrix operator generating the system (45) when it acts on the column vector containing twelve components of three "color" fields, in the basis $\left[\varphi_{+}, \chi_{+}, \psi_{+}, \varphi_{-}, \chi_{-}, \psi_{-}\right]$:

$$
E \mathbb{1}_{2} \otimes \mathbb{1}_{3} \otimes \mathbb{1}_{2}=m c^{2} \sigma_{3} \otimes B \otimes \mathbb{1}_{2}+\sigma_{1} \otimes Q_{3} \otimes c \sigma \cdot \mathbf{p}
$$

with energy and momentum operators on the left-hand side and the mass operator on the right-hand side:

$$
E \mathbb{1}_{2} \otimes \mathbb{1}_{3} \otimes \mathbb{1}_{2}-\sigma_{1} \otimes Q_{3} \otimes c \sigma \cdot \mathbf{p}=m c^{2} \sigma_{3} \otimes B \otimes \mathbb{1}_{2}
$$

Like with the standard Dirac equation, let us transform this equation so that the mass operator becomes proportional the the unit matrix. To do so, we multiply Equation (49) from the left by the matrix $\sigma_{3} \otimes B^{\dagger} \otimes \mathbb{1}_{2}$.

Now, we get the following equation, which enables us to interpret the energy and the momentum as the components of a Minkowskian four-vector $c p^{\mu}=[E, c \mathbf{p}]$ :

$$
E \sigma_{3} \otimes B^{\dagger} \otimes \mathbb{1}_{2}-i \sigma_{2} \otimes Q_{2} \otimes c \sigma \cdot \mathbf{p}=m c^{2} \mathbb{1}_{2} \otimes \mathbb{1}_{3} \otimes \mathbb{1}_{2},
$$


where we used the fact that under matrix multiplication, $\sigma_{3} \sigma^{3}=\mathbb{1}_{2}, B^{\dagger} B=\mathbb{1}_{3}$, and we define $Q_{2}=B^{\dagger} Q_{3}$.

The sixth power of this operator gives the same result as before,

$$
\left[E \sigma_{3} \otimes B^{\dagger} \otimes \mathbb{1}_{2}-i \sigma_{2} \otimes Q_{2} \otimes c \boldsymbol{\sigma} \cdot \mathbf{p}\right]^{6}=\left[E^{6}-c^{6} \mathbf{p}^{6}\right] \mathbb{1}_{12}=m^{6} c^{12} \mathbb{1}_{12}
$$

Equation (50) can be written in a concise manner using the Minkowskian indices and the usual pseudo-scalar product of two four-vectors as follows:

$$
\Gamma^{\mu} p_{\mu}=m c \mathbb{1}_{12} \text {, with } p^{0}=\frac{E}{c}, p^{k}=m c \frac{d x^{k}}{d s} .
$$

with $12 \times 12$ matrices $\Gamma^{\mu}, \quad(\mu=0,1,2,3)$ defined as follows:

$$
\Gamma^{0}=\sigma_{3} \otimes B^{\dagger} \otimes \mathbb{1}_{2}, \quad \Gamma^{k}=i \sigma_{2} \otimes Q_{2} \otimes \sigma^{k}
$$

\section{The Lagrangian}

While constructing the Lagrangian serving as the integrand in the variational principle leading to the color Dirac equations, we shall follow the usual approach known from the classical Dirac formalism. In order to ensure the right properties under the Lorentz transformations and the positivity of energy (the zeroth component of the conserved four-current), a special "charge conjugation" of Dirac spinors is introduced, namely $\bar{\psi}=\psi^{\dagger} \gamma^{0}$.

The matrix $\gamma^{0}$ is its own inverse, so that the expression $\bar{\psi} \gamma^{0} \psi$ becomes positive definite, as:

$$
\bar{\psi} \gamma^{0} \psi=\left(\psi^{\dagger} \gamma^{0}\right) \gamma^{0} \psi=\psi^{\dagger}\left(\gamma^{0} \gamma^{0}\right) \psi=\psi^{\dagger} \psi
$$

because $\gamma^{0} \gamma^{0}=\mathbb{1}_{4}$. The Lagrangian of the Dirac field is then:

$$
\mathcal{L}_{\text {Dirac }}=\frac{i}{2}\left[\bar{\psi} \gamma^{\mu} \partial_{\mu} \psi-\partial_{\mu} \bar{\psi} \gamma^{\mu} \psi\right]-m^{2} \bar{\psi} \psi
$$

Now, we have to implement the same construction with our $12 \times 12$ generalized $\Gamma$-matrices, among which $\Gamma^{0}=\sigma_{3} \otimes B^{\dagger} \otimes \mathbb{1}_{2}$. The "color Dirac spinor" $\Psi$ is composed of six Pauli spinors $\left[\varphi_{+}, \chi_{+}, \psi_{+}, \varphi_{-}, \chi_{-}, \psi_{-}\right]^{T}$ (it is a column, but we use this notation to spare the space). Its Hermitian conjugate is a row: $\Psi^{\dagger}=\left[\varphi_{+}^{*}, \chi_{+}^{*}, \psi_{+}, \varphi_{-}^{*}, \chi_{-}^{*}, \psi_{-}^{*}\right]$, where the star $*$ denotes the complex conjugate.

In the matrix $\Gamma^{0}$, the factors $\sigma_{3}$ and $\mathbb{1}_{2}$ are their own inverses, but the inverse of the middle factor of the tensor product, the matrix $B^{\dagger}$, is its Hermitian conjugate, i.e., the matrix $B$. This is why we should define the "color Dirac charge conjugation" as follows:

$$
\bar{\Psi}=\Psi^{\dagger}\left(\Gamma^{0}\right)^{\dagger}=\Psi^{\dagger} \sigma_{3} \otimes B \otimes \mathbb{1}_{2}
$$

With the generalized charge conjugation so defined, we can write down the Lagrangian for the color Dirac field:

$$
\mathcal{L}_{\text {color }}=\frac{i}{2}\left[\bar{\Psi} \Gamma^{\mu} \partial_{\mu} \Psi-\partial_{\mu} \bar{\Psi} \Gamma^{\mu} \Psi\right]-m^{2} \bar{\Psi} \Psi .
$$

Explicitly, we have $\bar{\Psi}=\left[\varphi_{+}^{*}, \chi_{+}^{*}, \psi_{+}^{*},-\varphi_{-}^{*},-j \chi_{-}^{*},-j^{2} \psi_{-}^{*}\right]$, so that now, like in the case of the usual Dirac equation, the expression $\bar{\Psi} \Gamma^{0} \Psi$ is positive-definite:

$$
\bar{\Psi} \Gamma^{0} \Psi=\varphi_{+}^{*} \varphi_{+}+\chi_{+}^{*} \chi_{+}+\psi *_{+} \psi_{+}+\varphi_{-}^{*} \varphi_{-}+\chi_{-}^{*} \chi_{-}+\psi_{-}^{*} \psi_{-}=J^{0},
$$


the same result as if we added up similar scalar products of three separate Dirac spinors $\varphi, \chi$, and $\psi$, each composed of two Pauli spinors, e.g., $\varphi=\left(\begin{array}{c}\varphi_{+} \\ \varphi_{-}\end{array}\right)$, but in our case, the six Pauli spinors do not form three separate Dirac spinors, but are entangled with the $Z_{3}$ cyclic group imprinting the extra "color" symmetry. As in the usual case, the field equations are obtained by applying the variational principle to the action integral:

$$
\delta \int \sqrt{|\operatorname{detg}|} \mathcal{L}_{\text {color }} \mathrm{d}^{4} x
$$

treating $\bar{\Psi}$ and $\Psi$ as independent variables. We get, as expected,

$$
\Gamma^{\mu} p_{\mu} \Psi=m c \Psi, \quad\left(p_{\mu} \bar{\Psi}\right) \Gamma^{\mu}=-m c \Psi .
$$

From this, we get the conservation of the four-current $J^{\mu}=\bar{\Psi} \Gamma^{\mu} \Psi$ :

$$
\partial_{\mu} J^{\mu}=\partial_{\mu} \bar{\Psi} \Gamma^{\mu} \Psi+\bar{\Psi} \Gamma^{\mu} \partial_{\mu} \Psi=0 .
$$

Explicitly, the four components of the current are real Hermitian, and "colorless", or "white", because the three color components contribute equally; the zeroth component $\left(J^{0}\right)$ was already explicated above, and the space-like components read:

$$
J^{k}=\left(\varphi_{+}^{*} \sigma^{k} \chi_{-}+\varphi_{-}^{*} \sigma^{k} \chi_{+}+\chi_{+}^{*} \sigma^{k} \psi_{-}+\chi_{-}^{*} \sigma^{k} \psi_{+}+\psi_{+}^{*} \sigma^{k} \varphi_{-}+\psi_{-}^{*} \sigma^{k} \varphi_{+}\right)
$$

The energy-momentum tensor can be defined either canonically:

$$
T_{v}^{\mu}=\frac{\delta \mathcal{L}}{\partial_{\mu} \Psi^{\alpha}} \partial_{\nu} \Psi^{\alpha}-\delta_{v}^{\mu} \mathcal{L}
$$

where the index $\alpha=1,2, \ldots, 12$ runs across all the components of the generalized "color" Dirac spinor, or in the symmetric version, as $T_{\mu \nu}^{s y m}=\delta \mathcal{L} / \delta g^{\mu \nu}$.

\section{Gauge Fields of the Standard Model}

Minimal interaction with generalized gauge fields should follow the classical case, where the influence of an electromagnetic field on the Dirac particle was encoded in the gauge potential $A_{\mu}$ inserted in the usual way:

$$
\mathcal{L}=\bar{\psi} \gamma^{\mu}\left(p_{\mu}-e A_{\mu}\right) \psi
$$

Now, we should write:

$$
\mathcal{L}_{\text {color }}=\bar{\Psi} \Gamma^{\mu}\left(p_{\mu}-C \mathcal{A}_{\mu}\right) \Psi,
$$

where $C$ symbolizes a generalization of the electric charge, here possibly including other coupling constants.

It is not difficult to imagine the most general form of the "big" potential $\mathcal{A}_{\mu}$. In order to couple to our "colored" 12-component spinors, constructed out of six Pauli spinors, the potential operator $\mathcal{A}$ should have its values in $12 \times 12$ matrices. In order to separate gauge fields generated by three separate symmetries induced by three discrete groups $Z_{2} \times Z_{3} \times Z_{2}$, these matrices must display a similar tensor product structure. This means that three separate sectors should interact with their own gauge fields.

The three contributions to the global gauge potential $\mathcal{A}$ sum up therefore as follows:

$$
\mathcal{A}_{\mu}=\sigma_{k} A_{\mu}^{k} \otimes \mathbb{1}_{3} \otimes \mathbb{1}_{2}+\mathbb{1}_{2} \otimes \lambda_{a} B_{\mu}^{a} \otimes \mathbb{1}_{2}+\mathbb{1}_{2} \otimes \mathbb{1}_{3} \otimes A_{\mu}^{e m} \mathbb{1}_{2}
$$


One recognizes easily the three ingredients of the standard model's gauge fields:

The $S U(2)$ weak interaction potential $A_{\mu}^{i}$ aligned along the three $\sigma$-matrices of the first tensorial factor:

$$
\sigma_{k} A_{\mu}^{k} \otimes \mathbb{1}_{3} \otimes \mathbb{1}_{2}, \quad i, k, . .=1,2,3 .
$$

The $S U(3)$ strong interaction gauge potential is aligned on the $S U(3)$ Lie algebra matrix basis $\lambda_{a}$ :

$$
B_{\mu}=\mathbb{1}_{2} \otimes \lambda_{a} B_{\mu}^{a} \otimes \mathbb{1}_{2}, \quad, a, b=1,2, \ldots 8 .
$$

appearing as the second factor in the tensor product, and finally, the electromagnetic potential $A_{\mu}^{e m}$ aligned along the unit $2 \times 2$ matrix appearing as the third factor in the tensor product:

$$
\mathbb{1}_{2} \otimes \mathbb{1}_{3} \otimes A_{\mu} \mathbb{1}_{2}
$$

Three coupling constants should appear in front of the three gauge field contributions to the unified gauge potential:

$$
\mathcal{A}_{\mu}=f \sigma_{k} A_{\mu}^{k} \otimes \mathbb{1}_{3} \otimes \mathbb{1}_{2}+g \mathbb{1}_{2} \otimes \lambda_{a} B_{\mu}^{a} \otimes \mathbb{1}_{2}+e \mathbb{1}_{2} \otimes \mathbb{1}_{3} \otimes A_{\mu}^{e m} \mathbb{1}_{2},
$$

where $f$ standsfor the weak interaction coupling constant, $g$ for the strong interaction coupling constant, and $e$ is the elementary electric charge, all in appropriate units. The separation of three interaction sectors remains when we form the gauge-invariant expression, defining the field strength $\mathcal{F}_{\mu \nu}$ :

$$
\mathcal{F}_{\mu \nu}=\partial_{\mu} \mathcal{A}_{v}-\partial_{\nu} \mathcal{A}_{\mu}+\left[\mathcal{A}_{\mu}, \mathcal{A}_{\nu}\right] .
$$

where the $12 \times 12$ matrix valued tensor $\mathcal{F}_{\mu v}$ splits naturally into three independent parts:

$$
\begin{gathered}
F_{\mu \nu}^{k}=\partial_{\mu} A_{v}^{k}-\partial_{\nu} A_{\mu}^{k}+f \epsilon_{l m}^{k} A_{\mu}^{l} A_{\nu}^{m} \\
G_{\mu \nu}^{a}=\partial_{\mu} B_{v}^{a}-\partial_{\nu} B_{\mu}^{a}+g f_{b c}^{a} B_{\mu}^{b} B_{v}^{c} \\
F_{\mu \nu}=\partial_{\mu} A_{\nu}^{e m}-\partial_{\nu} A_{\mu}^{e m}
\end{gathered}
$$

representing respectively the field tensors of weak, strong, and electromagnetic interactions. The structure constants of the $S U(2)$ Lie algebra are denoted by $\epsilon_{l m}^{k}, k, l_{\ldots}=1,2,3$, andthose of the $S U$ (3) Lie algebra are denoted by $f_{b c}^{a}, a, b \ldots=1,2, \ldots, 8$. The absence of mixed terms in the quadratic parts (the commutators) is due to the fact that the three $12 \times 12$ matrices bearing the three potentials do commute with each other, so that only the homogeneous commutators remain different from zero.

The gauge field Lagrangian can be defined as the trace of the square of the field tensor:

$$
\mathcal{L}_{F}=-\frac{1}{4} \operatorname{Tr}\left[\mathcal{F}_{\mu v} \mathcal{F}^{\mu v}\right]
$$

Here again, the mixed terms do not contribute to the final expression, because only squares of the matrices representing various fields give rise to the non-vanishing trace, while all other products of matrices have zero trace, and we get explicitly:

$$
\mathcal{L}_{F}=-\frac{12}{4} F_{\mu \nu}^{k} F_{k}^{\mu v}-\frac{1}{4} G_{\mu v}^{a} G_{b}^{\mu v}-\frac{12}{4} F_{\mu v} F^{\mu v}
$$




\section{The $Z_{3}$ Lorentz Invariance}

Although the four $12 \times 12$ matrices do not satisfy the usual anti-commutation relations similar to those of the $4 \times 4$ Dirac matrices $\gamma^{\mu}$, i.e., $\gamma^{\mu} \gamma^{\nu}+\gamma^{v} \gamma^{\mu}=2 g^{\mu v} \mathbf{1}_{4}$, nevertheless, the system of equations satisfied by the 12-dimensional wave function $\Psi$,

$$
-i \hbar \Gamma^{\mu} \partial_{\mu} \Psi=m c \Psi
$$

is a hyperbolic one, and has the same light cone as the Klein-Gordon equation. To corroborate this statement, let us first consider the massless case, $-i \hbar \Gamma^{\mu} \partial_{\mu} \Psi=0$.

Assuming the general solution of the form $e^{k_{\mu} x^{\mu}}$, we can replace the derivations by the components of the wave four-vector $k^{\mu}$ and take the sixth power of the matrix $\Gamma^{\mu} k_{\mu}$. The resulting dispersion relation is shown to be:

$$
\begin{gathered}
k_{0}^{6}-|\mathbf{k}|^{6}=\left(k_{0}^{2}-|\mathbf{k}|^{2}\right)\left(k_{0}^{2}-j|\mathbf{k}|^{2}\right)\left(k_{0}^{2}-j^{2}|\mathbf{k}|^{2}\right)= \\
=\left(k_{0}^{2}-|\mathbf{k}|^{2}\right)\left(k_{0}^{4}+k_{0}^{2}|\mathbf{k}|^{2}+|\mathbf{k}|^{4}\right)=0 .
\end{gathered}
$$

The first factor defines the usual light cone, while the factor of degree four is strictly positive (besides the origin zero). The system has only one characteristic surface, which is the same for all massless fields. Each of the three factors remains invariant under a different representation of the $S L(2, \mathrm{C})$ group. Let us introduce three matrices representing the same four-vector $k^{\mu}$ :

$$
K_{3}=\left(\begin{array}{ll}
k_{0} & k_{x} \\
k_{x} & k_{0}
\end{array}\right), \quad K_{1}=\left(\begin{array}{cc}
k_{0} & j k_{x} \\
j k_{x} & k_{0}
\end{array}\right), \quad K_{2}=\left(\begin{array}{cc}
k_{0} & j^{2} k_{x} \\
j^{2} k_{x} & k_{0}
\end{array}\right) \text {, }
$$

whose determinants are, respectively,

$$
\operatorname{det} K_{1}=k_{0}^{2}-j^{2} k_{x}^{2}, \quad \operatorname{det} K_{2}=k_{0}^{2}-j k_{x}^{2}, \operatorname{det} K_{3}=k_{0}^{2}-k_{x}^{2} .
$$

Note that only the third matrix $K_{3}$ is Hermitian and corresponds to a real space-time vector $k^{\mu}$, while neither of the remaining two matrices $K_{1}$ and $K_{2}$ is Hermitian; however, one is the Hermitian conjugate of another.

In what follows, we shall replace the absolute value of the wave vector $|\mathbf{k}|$ by a single spatial component, say $k_{x}$, because for any given four-vector $k^{\mu}=\left[k_{0}, \mathbf{k}\right]$, we can choose the coordinate system in such a way that its $x$-axis should be aligned along the vector $\mathbf{k}$. Then, it is easy to check that one has:

$$
\begin{gathered}
\left(\begin{array}{cc}
\cosh u & \sinh u \\
\sinh u & \cosh u
\end{array}\right)\left(\begin{array}{c}
k_{0} \\
k_{x}
\end{array}\right)=\left(\begin{array}{l}
k_{0}^{\prime} \\
k^{\prime}{ }_{x}
\end{array}\right) \\
\left(\begin{array}{cc}
\cosh u & j^{2} \sinh u \\
j \sinh u & \cosh u
\end{array}\right)\left(\begin{array}{c}
k_{0} \\
j k_{x}
\end{array}\right)=\left(\begin{array}{c}
k_{0}^{\prime} \\
j k_{x}^{\prime}
\end{array}\right), \quad\left(\begin{array}{cc}
\cosh u & j \sinh u \\
j^{2} \sinh u & \cosh u
\end{array}\right)\left(\begin{array}{c}
k_{0} \\
j^{2} k_{x}
\end{array}\right)=\left(\begin{array}{c}
k_{0}^{\prime} \\
j^{2} k_{x}^{\prime}
\end{array}\right) .
\end{gathered}
$$

The transformed vectors are given by the following expressions:

(i) $k_{0}^{\prime}=k_{0} \cosh u+k_{x} \sinh u, \quad k_{x}^{\prime}=k_{0} \sinh u+k_{x} \cosh u$

(ii) $k_{0}^{\prime}=k_{0} \cosh u+j^{2} k_{x} \sinh u, \quad k_{x}^{\prime}=j k_{0} \sinh u+k_{x} \cosh u$

(iii) $k_{0}^{\prime}=k_{0} \cosh u+j k_{x} \sinh u, \quad k_{x}^{\prime}=j^{2} k_{0} \sinh u+k_{x} \cosh u$ 
Let us now introduce a $6 \times 6$ matrix composed of the above three $2 \times 2$ matrices:

$$
\left(\begin{array}{ccc}
0 & k_{0} \mathbb{1}_{2}+\mathbf{k} \cdot \sigma & 0 \\
0 & 0 & k_{0} \mathbb{1}_{2}+j \mathbf{k} \cdot \sigma \\
k_{0} \mathbb{1}_{2}+j^{2} \mathbf{k} \cdot \sigma & 0 & 0
\end{array}\right)
$$

or, more explicitly,

$$
K=\left(\begin{array}{cccccc}
0 & 0 & k_{0} & k_{x} & 0 & 0 \\
0 & 0 & k_{x} & k_{0} & 0 & 0 \\
0 & 0 & 0 & 0 & k_{0} & j k_{x} \\
0 & 0 & 0 & 0 & j k_{x} & k_{0} \\
k_{0} & j^{2} k_{x} & 0 & 0 & 0 & 0 \\
j^{2} k_{x} & k_{0} & 0 & 0 & 0 & 0
\end{array}\right)
$$

It is easy to check that:

$$
\operatorname{det} K=\left(\operatorname{det} K_{1}\right) \cdot\left(\operatorname{det} K_{2}\right) \cdot\left(\operatorname{det} K_{3}\right)=\left(k_{0}^{2}-k_{x}^{2}\right)\left(k_{0}^{2}-j^{2} k_{x}^{2}\right)\left(k_{0}^{2}-j k_{x}^{2}\right)=k_{0}^{6}-k_{x}^{6} .
$$

The eigenvalues (which all have multiplicity two) of this operator are easily found to be:

$$
\pm\left(k_{0}^{6}-k_{x}^{6}\right)^{\frac{1}{6}}, \quad \pm j\left(k_{0}^{6}-k_{x}^{6}\right)^{\frac{1}{6}}, \pm j^{2}\left(k_{0}^{6}-k_{x}^{6}\right)^{\frac{1}{6}},
$$

It is also remarkable that the determinant remains the same in the basis in which the ternary Dirac operator was proposed, namely when we consider the matrix:

$$
K=\left(\begin{array}{cccccc}
k_{0} & 0 & 0 & k_{x} & 0 & 0 \\
0 & k_{0} & k_{x} & 0 & 0 & 0 \\
0 & 0 & k_{0} & 0 & 0 & j k_{x} \\
0 & 0 & 0 & k_{0} & j k_{x} & 0 \\
0 & j^{2} k_{x} & 0 & 0 & k_{0} & 0 \\
j^{2} k_{x} & 0 & 0 & 0 & 0 & k_{0}
\end{array}\right)
$$

Let us show now that the spinorial representation of Lorentz boosts can be applied to each of the three matrices $K_{1}, K_{2}$, and $K_{3}$ separately, keeping their determinants unchanged. As a matter of fact, besides the well-known formula:

$$
\left(\begin{array}{ll}
\cosh \frac{u}{2} & \sinh \frac{u}{2} \\
\sinh \frac{u}{2} & \cosh \frac{u}{2}
\end{array}\right)\left(\begin{array}{ll}
k_{0} & k_{x} \\
k_{x} & k_{0}
\end{array}\right) \quad\left(\begin{array}{ll}
\cosh \frac{u}{2} & \sinh \frac{u}{2} \\
\sinh \frac{u}{2} & \cosh \frac{u}{2}
\end{array}\right)=\left(\begin{array}{ll}
k_{0}^{\prime} & k_{x}^{\prime} \\
k_{x}^{\prime} & k_{0}^{\prime}
\end{array}\right),
$$

with:

$$
k_{0}^{\prime}=k_{0} \cosh u+k_{x} \sinh u, \quad k_{x}^{\prime}=k_{0} \sinh u+k_{x} \cosh u .
$$

which becomes apparent when we remind that:

$$
\cosh ^{2} \frac{u}{2}+\sinh ^{2} \frac{u}{2}=\cosh u \text { and } 2 \sinh \frac{u}{2} \cosh \frac{u}{2}=\sinh u,
$$

keeping unchanged the Minkowskian scalar product: $k_{0}^{\prime 2}-k_{x}^{\prime 2}=k_{0}^{2}-k_{x}^{2}$, we have also two transformations of the same kind which keep invariant the "complexified" Minkowskian squares appearing as factors in the sixth-order expression $k_{0}^{6}-k_{x}^{6}$, namely:

$$
k_{0}^{2}-j k_{x}^{2} \text { and } k_{0}^{2}-j^{2} k_{x}^{2} \text {. }
$$


The above expressions can be identified as the determinants of the following $2 \times 2$ matrices:

$$
k_{0}^{2}-j k_{x}^{2}=\operatorname{det}\left(\begin{array}{cc}
k_{0} & j^{2} k_{x}^{2} \\
j^{2} k_{x} & k_{0}
\end{array}\right), \quad k_{0}^{2}-j^{2} k_{x}^{2}=\operatorname{det}\left(\begin{array}{cc}
k_{0} & j k_{x}^{2} \\
j k_{x} & k_{0}
\end{array}\right) .
$$

It is easy to check that we have:

$$
\left(\begin{array}{cc}
\cosh \frac{u}{2} & \sinh \frac{u}{2} \\
\sinh \frac{u}{2} & \cosh \frac{u}{2}
\end{array}\right)\left(\begin{array}{cc}
k_{0} & j k_{x} \\
j k_{x} & k_{0}
\end{array}\right)\left(\begin{array}{cc}
\cosh \frac{u}{2} & \sinh \frac{u}{2} \\
\sinh \frac{u}{2} & \cosh \frac{u}{2}
\end{array}\right)=\left(\begin{array}{cc}
k_{0}^{\prime} & j k^{\prime}{ }_{x} \\
j k_{x}^{\prime} & k_{0}^{\prime}
\end{array}\right),
$$

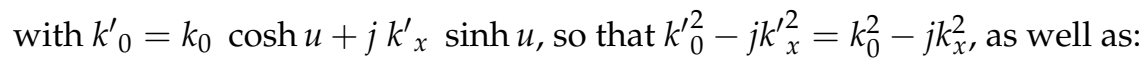

$$
\left(\begin{array}{cc}
\cosh \frac{u}{2} & \sinh \frac{u}{2} \\
\sinh \frac{u}{2} & \cosh \frac{u}{2}
\end{array}\right)\left(\begin{array}{cc}
k_{0} & j^{2} k_{x} \\
j^{2} k_{x} & k_{0}
\end{array}\right)\left(\begin{array}{cc}
\cosh \frac{u}{2} & \sinh \frac{u}{2} \\
\sinh \frac{u}{2} & \cosh \frac{u}{2}
\end{array}\right)=\left(\begin{array}{cc}
k_{0}^{\prime} & j^{2} k_{x}^{\prime} \\
j^{2} k_{x}^{\prime} & k_{0}^{\prime}
\end{array}\right),
$$

The bottom line is the following: the $12 \times 12$ matrix formed by the tensor product of $\sigma_{3}$ with the $6 \times 6$ matrix $K$ defined above has the same determinant and the same eigenvalues (73) and (74) as the generalized Dirac operator (49), if we replace $k_{0}$ by $E$ and $\mathbf{k}$ by $c \mathbf{p}$. We have shown that the determinant of the matrix $\sigma_{3} \otimes K$ (equal to $\left.\left(k_{0}^{6}-k_{x}^{6}\right)^{2}\right)$ remains invariant under the generalized Lorentz transformation composed of three representations, the usual unitary one and two complex ones. Therefore, there exists a similarity between the two matrices, which preserves the invariance under the generalized Lorentz group intertwined with $Z_{3}$.

More details can be found in Refs. [17,18]; in particular, the basis of general solutions of the sixth-order generalization of Dirac's equation is presented, as well as their propagating cubic combinations. The presence of six poles in the propagator whose Fourier image is proportional to the inverse of the expression $k_{0}^{6}-|\mathbf{k}|^{6}-M^{6}$ has similar consequences on the behavior of solutions as what is known from the Lee-Wick model [19,20], as discussed in Ref. [21], also displaying six poles, but not in a Lorentz-covariant manner.

Funding: This research received no external funding.

Acknowledgments: The author wishes to express his gratitude to Michel Dubois-Violette, Rafał Abłamowicz, Jan-Willem van Holten, Yakov Krasnov, Jerzy Lukierski, and Mo-Lin Ge for many interesting discussions and enlightening advice and remarks.

Conflicts of Interest: The author declares no conflicts of interest.

\section{References}

1. Born, M.; Jordan, P. Zur quantenmechanik. Z. Phys. 1925, 34, 858-878. [CrossRef]

2. Von Neumann, J. Mathematical Foundations of Quantum Mechanics; Princeton University Press: Princeton, NJ, USA, 1996.

3. Dubois-Violette, M.; Kerner, R.; Madore, J. Noncommutative differential geometry of matrix algebras. J. Math. Phys. 1990, 31, 316-322. [CrossRef]

4. Dubois-Violette, M.; Kerner, R.; Madore, J. Noncommutative geometry and new models of gauge theories. J. Math. Phys. 1990, 31, 323-331. [CrossRef]

5. Froggatt, C.D.; Nielsen, H.B. Origin of Symmetries; World Scientific: Singapore, 1991.

6. Pauli, W. The Connection Between Spin and Statistics. Phys. Rev. 1940, 58, 716-722. [CrossRef]

7. Dyson, F.J. Ground State Energy of a Finite System of Charged Particles. J. Math. Phys. 1967, 8, 1538-1545. [CrossRef]

8. Dyson, F.J.; Lenard, A. Stability of matter. II. J. Math. Phys. 1968, 9, 698-711.

9. Streater, R.F.; Wightman, A.S. PCT, Spin ans Statistics and All That; W.A. Benjamin, Inc.: New York, NY, USA; Amsterdam, The Netherlands, 1964.

10. Dirac, P.A.M. The Principles of Quantim Mechanics, 4th ed.; Oxford University Press: Oxford, UK, 1958. 
11. Bjorken, J.D.; Drell, D.S. Relativistic Quantum Mechanics; McGraw-Hill: London, UK; New York, NY, USA, 1964.

12. Gell-Mann, M.; Ne'eman, Y. The Eightfold Way; Benjamin: New York, NY, USA, 1964.

13. Lipkin, H.J. Frontiers of the Quark Model; pr. WIS-87-47-PH; Weizmann Institute: Rehovot, Israel, 1987.

14. Okubo, S. Triple products and Yang-Baxter equation. I. Octonionic and quaternionic triple systems. J. Math. Phys. 1993, 34, 3273-3291. [CrossRef]

15. Kerner, R. Graduation $Z_{3}$ et la racine cubique de l'opérateur de Dirac. C. R. Acad. Sci. Paris 1991, 312, $191-196$.

16. Kerner, R. $Z_{3}$ graded algebras and the cubic root of the supersymmetry translations. J. Math. Phys. 1992, 33, 403-411. [CrossRef]

17. Kerner, R. Ternary Generalization of Pauli's Principle and the Z6-Graded Algebras. Phys. At. Nucl. 2017, 80, 522-534. [CrossRef]

18. Kerner, $R$. Ternary $Z_{2}$ and $Z_{3}$ graded algebras and color dynamics. In Mathematical Structures and Applications; Springer: New York, NY, USA, 2018; pp. 311-357.

19. Lee, T.D.; Wick, G.C. Negative metric and the unitarity of the S-matrix. Nucl. Phys. B 1969, 9, $209-243$. [CrossRef]

20. Lee, T.D.; Wick, G.C. Finite theory of quantum electrodynamics. Phys. Rev. D 1970, 2, 1033. [CrossRef]

21. Anselmi, D.; Piva, M. Perturbative unitarity of Lee-Wick quantum field theory. Phys. Rev. D 2017, 96, 045009. [CrossRef]

(C) 2018 by the authors. Licensee MDPI, Basel, Switzerland. This article is an open access article distributed under the terms and conditions of the Creative Commons Attribution (CC BY) license (http:/ / creativecommons.org/licenses/by/4.0/). 